\title{
Measures of Specificity of Fuzzy Sets Under T-Indistinguishabilities
}

\author{
Luis Garmendia, Ronald R. Yager, Enric Trillas, and Adela Salvador
}

\begin{abstract}
The concept of measure of specificity of fuzzy sets is extended in this paper to measure of specificity under the knowledge of T-indistinguishabilities. Four axioms of measure of specificity under T-indistinguishabilities are given. An algorithm to compute the inference independent set $\Im$ from a fuzzy set and a T-indistinguishability is used to measure the specificity of fuzzy sets under $\mathbf{T}$-indistinguishabilities satisfying the axioms.
\end{abstract}

Index Terms-Measure of specificity under similarity, measure of specificity under T-indistinguishability, specificity, T-indistinguishability.

\section{INTRODUCTION}

$\mathbf{T}$ HE measures of specificity under similarities are introduced as a solution for "the jacket problem" [5]. When some knowledge is given by a similarity, the utility of some nonspecific information is increased. For example, if it is known that the temperature is in an interval, it is not itself specific information, but it can be specific and useful in order to choose which jacket to wear today. In general, when our knowledge is increased by a given similarity, then the specificity of fuzzy sets is also increased. This paper studies a first approach of this problem when the existing knowledge is increased by a T-indistinguishability for any $\mathrm{t}$-norm $\mathrm{T}$.

Any $\alpha$-cut of a similarity relation is a classical equivalence relations, so given a fuzzy set $\mu$ on a finite space $X$ and a similarity relation, it is possible to make a partition of $X$ for each value $\alpha$ in $[0,1]$. This fact is used to define measures of specificity under similarities [5]. However, the $\alpha$-cuts of a T-indistinguishability are not necessarily classical equivalence relations, so new concepts, axioms, definitions and methods are to be introduced to solve this problem.

The axioms of measures of specificity under T-indistinguishabilities given in this paper generalise and extends the previous concept of measures of specificity under similarities.

Manuscript received July 30, 2004; revised September 12, 2005 and January 3, 2006. This work was supported in part by the Spanish MCyT project BFM2002-00281.

L. Garmendia is with Facultad de Informática, Departamento de Lenguajes y Sistemas Informáticos, Universidad Complutense of Madrid, 28040 Madrid, Spain (e-mail: lgarmend@fdi.ucm.es).

R. R. Yager is with Iona College, New Rochelle, NY 10801 USA (e-mail: yager@panix.com)

E. Trillas is with the Facultad de Informática, Departamento de Inteligencia Artificial, Technical University of Madrid, Madrid 28668, Spain (e-mail: etrillas@fi.upm.es)

A. Salvador is with the Departamento de Matemática Aplicada, E.T.S.I. Caminos,Technical University of Madrid, Madrid 28040, Spain (e-mail: ma09@caminos.upm.es)

Digital Object Identifier 10.1109/TFUZZ.2006.876731
An algorithm to compute a measure of specificity under T-indistinguishabilities is given, and it is proved that the output measure verifies the axioms of measures of specificity under T-indistinguishabilities.

\section{PRELIMINARIES}

Let $X$ be a finite set of elements.

\section{A. Definition 2.1: Measure of Specificity}

Let $[0,1]^{X}$ be the set of fuzzy sets on $X$. A measure of specificity [4], [1], [2] is a function $S p:[0,1]^{X} \rightarrow[0,1]$ such that

1) $\mathrm{Sp}(\mu)=1$ if and only if $\mu$ is a singleton $(\mu=\{\mathrm{x}\})$;

2) $\operatorname{Sp}(\emptyset)=0$;

3) if $\mu$ and $\eta$ are normal fuzzy sets in $X$ and $\mu \subset \eta$, then $\mathrm{Sp}$ $(\mu) \geq \operatorname{Sp}(\eta)$.

The first condition establishes that only crisp sets with just one element (singletons) have the maximum specificity. The second condition assumes that the empty set have minimum specificity. Note that other non-empty fuzzy sets could also have specificity zero. The third condition imposes that the specificity measure of a normal fuzzy set is lower when the membership degree of its elements are increased. A fuzzy set $\mu$ on $X$ is normal if there is an element $x \in X$ such that $\mu(x)=1$.

The "jacket problem" [5] introduce the concept of specificity of fuzzy sets under similarities. "The weather is over $20^{\circ} \mathrm{C}$ " is not a specific information, but it is indeed specific in the problem of choosing the right jacket to wear. Some temperature ranges could be similar or indistinguishable in order to make a good decision to choose a jacket to wear.

A fuzzy relation is a T-indistinguishability if it is reflexive, symmetric and T-transitive. A fuzzy relation $R: X \times X \rightarrow$ $[0,1]$ is T-transitive if and only if $T(R(a, b), R(b, c)) \leq R(a, c)$ for all $a, b, c$ in $X$. When $\mathrm{T}$ is the t-norm minimum, then the indistinguishability is a similarity.

The $\alpha$-cut of a similarity $S$ is a classic equivalence relation denoted $S_{\alpha}$. Let $\pi_{\alpha}$ be the set of equivalence classes of $X$ by the relation $S_{\alpha}$. Let $\mu_{\alpha} / S$ be the set of equivalence classes of $\pi_{\alpha}$ defined as follows: A class $\pi_{\alpha}(i)$ belongs to $\mu_{\alpha} / S$ if there exists an element $\mathrm{x}$ contained in $\pi_{\alpha}(i)$ and in the $\alpha$-cut of $\mu$.

The measure of specificity of a fuzzy set $\mu$ under a similarity $S[5]$ is computed as

$$
S_{p}(\mu / S)=\int_{0}^{\alpha_{\max }} \frac{1}{\operatorname{Card}\left(\mu_{\alpha} / S\right)} d \alpha .
$$

The measure of specificity under a similarity is maximal when $\mu_{\alpha}$ is contained in one class of $S_{\alpha}$ for all $\alpha$ in $[0,1]$. 
The given expression is computable when $S$ is a similarity, but it is not valid when $S$ is any T-indistinguishability, because when $\mathrm{T}$ is not the minimum t-norm, all the $\alpha$-cuts of $S$ are not equivalence relations and then $\mu_{\alpha} / S$ is not well defined.

\section{AXIOMS OF MEASURES OF SPECIFICITY UNDER A T-INDISTINGUISHABILITY}

\section{A. Definition 3.1}

Let $X$ be a crisp finite set, let $\mu$ be a fuzzy set or a possibility distribution on $X$, let $S: X \times X \rightarrow[0,1]$ be a T-indistinguishability and let $\mathrm{Sp}$ be a measure of specificity on fuzzy sets.

$S p_{S}(\mu)$ is a measure of specificity under the T-indistinguishability $\boldsymbol{S}$ when it verifies the following axioms:

1) $S p_{S}(\{x\})=1$;

2) $S p_{S}(\emptyset)=0$

3) $S p_{I d}(\mu)=S p(\mu)$;

4) $S p_{S}(\mu) \geq S p(\mu)$.

The first axiom stands that the measures of specificity of a singleton under any T-indistinguishability is always one.

The second axiom extends the second condition of measure of specificity [4].

The empty set does not provide any information, even if a T-indistinguishability is known, so the specificity of the empty set is zero.

The third axiom stands that a measure of specificity of a fuzzy set under the identity relation (Id) is the measure of specificity of the fuzzy set.

The identity relation is a classical equivalence relation that gives a class for each element in $X$, so the $\mathrm{T}$-indistinguishability does not provide any extra information.

The fourth axiom indicates that when the available information is increased with a T-indistinguishability, then the usability of the information contained in the fuzzy set is always equal or higher. For example, when $S$ is a classical equivalence relation then the election can be done choosing into a lower number of classes. Then a decision is easier and so the measure of specificity should be higher.

\section{Algorithm to COMPute An INFERENCE INDEPENDENT} SET

Definition 4.1: The Relation $\succeq$ : Let $\mu$ be a fuzzy set on a finite set $X=\left\{x_{1}, \ldots, x_{n}\right\}$, let T be a t-norm and let $S$ be a T-indistinguishability, then the "infers" relation $\succeq$ on $X \times X$ is defined by: $\mathbf{x}_{k} \succeq x_{j}$ if and only if $\left.T\left(\mu\left(x_{k}\right), S\left(x_{k}, x_{j}\right)\right)\right) \geq$ $\mu\left(x_{j}\right)$.

If $X_{k} \succeq X_{j}$ it is said that $X_{j}$ is inferred by $X_{k}$.

\section{A. Proposition 4.1}

The relation $\succeq$ is a classic preorder relation on $X$.

\section{B. Proof}

The $\succeq$ relation is reflexive:
$\left.T\left(\mu\left(x_{i}\right), S\left(x_{i}, x_{i}\right)\right)\right)=T\left(\mu\left(x_{i}\right), 1\right)=\mu\left(x_{i}\right), \operatorname{so} x_{i} \succeq x_{i}$.

The $\succeq$ relation is transitive.

Let us suppose that $x_{i} \succeq x_{j}$ and $x_{j} \succeq x_{k}$. Then,

$x_{i} \succeq x_{j}, \operatorname{soT}\left(\mu\left(x_{i}\right), S\left(x_{i}, x_{j}\right)\right) \geq \mu\left(x_{x}\right)$.

$x_{j} \succeq x_{k}, \operatorname{soT}\left(\mu\left(x_{j}\right), S\left(x_{j}, x_{k}\right)\right) \geq \mu\left(x_{k}\right)$.

Hence, $\mu\left(x_{k}\right) \leq T\left(\mu\left(x_{j}\right), S\left(x_{j}, x_{k}\right)\right)$

$\leq T\left(T\left(\mu\left(x_{i}\right), S\left(x_{i}, x_{j}\right)\right), S\left(x_{j}, x_{k}\right)\right)$ ( $T$ is associative $)$

$=T\left(\mu\left(x_{i}\right), T\left(S\left(x_{i}, x_{j}\right), S\left(x_{j}, x_{k}\right)\right)\right.$ (S is T-transitive)

$\leq T\left(\mu\left(x_{i}\right), S\left(x_{i}, x_{k}\right)\right)$

and so $x_{i} \succeq x_{k}$.

\section{Definition 4.2: Representative Element}

Let $\mu$ be a fuzzy set on a finite space $X=\left\{x_{1}, \ldots, x_{n}\right\}$, let $T$ be a t-norm and let $S$ be a T-indistinguishability. An element $x_{i}$ in $X$ is a representative element when if $x_{j} \succeq x_{i}$ then $j=i$.

So, a representative element cannot be inferred from other elements by making fuzzy inference using the t-norm $T$ with the fuzzy set and the T-indistinguishability.

\section{Definition 4.3: T-Inference Independent Set}

Let $\mu$ be a fuzzy set on a finite space $X=\left\{x_{1}, \ldots, x_{n}\right\}$, let $T$ be a t-norm and let $S$ be a T-indistinguishability.

An inference independent set $\Im$ is defined by a fuzzy set on $X$ such that if $x$ is a representative element then $\Im(x)=\mu(x)$ and, otherwise, $\Im(x)=0$.

Note that the inference independent set is a fuzzy set $\Im$ on $X$ in which only the representative elements have a positive membership degree.

\section{E. Algorithm}

Let $\mu$ be a fuzzy set on a finite space $X=\left\{x_{1}, \ldots, x_{n}\right\}$ sorted by their membership degrees. So $\mu\left(x_{1}\right) \geq \cdots \geq$ $\mu\left(x_{n}\right)$. Let $T$ be a t-norm and let $S$ be a T-indistinguishability on $X$.

The algorithm to compute the T-inference independent set $\Im$ is given in pseudocode as follows.

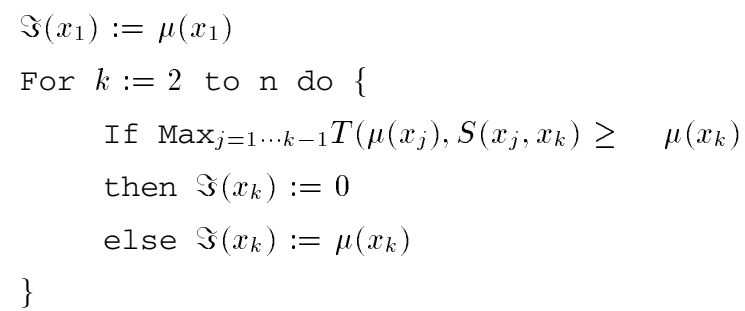

The algorithm purpose is to get rid in $\Im$ of the elements $x_{j}$ when it exits $a k, k \neq j$, such that $x_{k} \succeq x_{j}$. Those elements are "inferred" by others and then their membership degree in $\Im$ is zero.

\section{F. Example 4.1}

Let $\mu$ be a fuzzy set on $X=\left\{x_{1}, \ldots, x_{5}\right\}$ with the following membership degrees : $\mu=$ $1 / x_{1}, 0.7 / x_{2}, 0.5 / x_{3}, 0.2 / x_{4}, 0 / x_{5}$. 
Let $S$ be the following min-indistinguishability:

$$
S=\left(\begin{array}{ccccl}
1 & 1 & 0 & 0.5 & 0.2 \\
1 & 1 & 0 & 0.5 & 0.2 \\
0 & 0 & 1 & 0 & 0 \\
0.5 & 0.5 & 0 & 1 & 0.2 \\
0.2 & 0.2 & 0 & 0.2 & 1
\end{array}\right) .
$$

The algorithm is applied as follows to compute a min-inference independent set. $\Im\left(x_{1}\right):=\mu\left(x_{1}\right)=1$. 0.7

$k=2$ computes:

$\left.\left.\operatorname{Max}_{j=1}\left\{\operatorname{Min}\left(\mu\left(x_{j}\right), x_{2}\right)\right)\right)\right\}=\operatorname{Min}(1,1)=1 \geq \mu\left(X_{2}\right)=$

Then, $x_{2}$ is inferred by $x_{1}$, so $\Im\left(x_{2}\right):=0$.

$k=3$

$$
\begin{aligned}
& \operatorname{Max}_{j=1 \cdots 2}\left\{\operatorname{Min}\left(\mu\left(x_{j}\right), S\left(x_{j}, x_{3}\right)\right)\right\}= \\
&\operatorname{Max}\{\operatorname{Min}(1,0), \operatorname{Min}(0.7,0))\}=0<\mu\left(x_{3}\right)=0.5 .
\end{aligned}
$$

Then, $x_{3}$ is not inferred by $x_{1}$ nor $x_{2}$ so $\Im\left(x_{3}\right):=\mu\left(x_{3}\right)=$ 0.5 .

$$
k=4
$$

$\operatorname{Max}_{j=1 \ldots 3}\left\{\operatorname{Min}\left(\mu\left(x_{j}\right), S\left(x_{j}, x_{4}\right)\right)\right\}=\operatorname{Max}\{\operatorname{Min}$

$(1,0.5), \operatorname{Min}(0.7,0.5), \operatorname{Min}(0.5,0))\}=0.5>\mu\left(x_{4}\right)=0.2$.

Then, $x_{1} \succeq x_{4}, \operatorname{so\Im }\left(x_{4}\right):=0$.

$k=5$

$\operatorname{Max}_{j=1 \ldots 4}\left\{\operatorname{Min}\left(\mu\left(x_{j}\right), S\left(x_{j}, x_{5}\right)\right\}=\operatorname{Max}\{\operatorname{Min}(1,0.2)\right.$, $\operatorname{Min}(0.7,0.2), \operatorname{Min}(0.5,0), \operatorname{Min}\}(0.2,0.2)=0.2>\mu\left(x_{5}\right)=0$.

Then, $x_{1} \succeq x_{5}, s_{0}\left(x_{5}\right):=0$.

The fuzzy min-inference is $1 / x_{1}, 0 / x_{2}, 0.5 / x_{3}, 0 / x_{4}, 0 / x_{5}$.

\section{G. Example 4.2}

Let $T$ be the t-norm product.

Let $\mu$ be a fuzzy set on $X=x_{1}, \ldots, x_{5}$ with the following membership degrees: $\mu=$ $1 / x_{1}, 0.7 / x_{2}, 0.5 / x_{3}, 0.2 / x_{4}, 0 / x_{5}$.

Let $S$ be a prod-indistinguishability represented by

$$
S=\left(\begin{array}{ccccl}
1 & 0.25 & 0 & 0.5 & 0.2 \\
0.25 & 1 & 0 & 0.5 & 0.2 \\
0 & 0 & 1 & 0 & 0 \\
0.5 & 0.5 & 0 & 1 & 0.1 \\
0.2 & 0.2 & 0 & 0.1 & 1
\end{array}\right)
$$

Note that $S$ is not a similarity, because $S\left(x_{1}, x_{2}\right)=0.25<$ $\operatorname{Min} S\left(x_{1}, x_{4}\right), S\left(x_{4}, x_{2}\right)=\operatorname{Min} 0.5,0.5=0.5$.
The algorithm is applied as follows to compute a prod-inference independent set:

$$
\Im\left(x_{1}\right):=\mu(x)_{1}=1
$$

$k=2$

$\operatorname{Max}_{j=1}\left\{T\left(\mu\left(x_{j}\right), S\left(x_{j}, x_{2}\right)\right\}=T(1,0.25)=0.25<\mu\left(x_{2}\right)=0.7\right.$

So, $x_{2}$ is not inferred by other elements in $X$ and then it is a representative element $\Im\left(x_{2}\right):=\mu\left(x_{2}\right)=0.7$

$k=3$

$\operatorname{Max}_{j=1 \ldots 2}\left\{T\left(\mu\left(x_{j}\right), S\left(x_{j}, x_{3}\right)\right)\right\}$

$\operatorname{Max}\{T(1,0), T(0.7,0)\}=0<\mu\left(x_{3}\right)=0.5$.

Then, $x_{3}$ is not inferred by $x_{1}$ nor $x_{2}$ so $\Im\left(x_{3}\right):=\mu\left(x_{3}\right)=$ 0.5 .

$k=4$

$$
\begin{aligned}
\operatorname{Max}_{j=1 \ldots 3}\left\{T\left(\mu\left(x_{j}\right), S\left(x_{j}, x_{4}\right)\right)\right\}=\operatorname{Max}\{T(1,0.5), \\
T(0.7,0.5), T(0.5,0)\}=0.5>\mu\left(x_{4}\right)=0.2 .
\end{aligned}
$$

Then, $x_{1} \succeq x_{4}, \operatorname{so} \Im\left(x_{4}\right):=0$.

$k=5$

$$
\begin{aligned}
& \operatorname{Max}_{j=1 \ldots 4}\left\{T\left(\mu\left(x_{j}\right), S\left(x_{j}, x_{5}\right)\right)\right\}=\operatorname{Max}\{T(1,0.2), \\
& \quad T(0.7,0.2), T(0.5,0), T(0.2,0.1)\}=0.2>\mu\left(x_{5}\right)=0 .
\end{aligned}
$$

Then $x_{1} \succeq x_{5}, \operatorname{so\Im }\left(x_{5}\right):=0$.

The fuzzy prod-inference independent set $\Im$ is $1 / x_{1}, 0.7 / x_{2}, 0.5 / x_{3}, 0 / x_{4}, 0 / x_{5}$.

\section{Applaying the Algorithm to COMPUTE MEASURES OF SPECIFICITY OF FuZZy SETS UNDER T-INDISTINGUISHABILITIES}

Given a measure of specificity for fuzzy sets, it can be computed a measure of specificity of a fuzzy set under a T-indistinguishability as the measure of specificity of its inference independent set $\Im$.

\section{A. Theorem 5.1}

The measure of specificity of $\mu$ under a T-indistinguishability $S, S p_{S}(\mu)$, computed by $S p(\Im)$, satisfy the four axioms of measures of specificity under T-indistinguishabilities.

Proof: Let $\mu$ be a fuzzy set on a finite space $X=$ $\left\{x_{1}, \ldots, x_{n}\right\}$ sorted by their membership degree so that $\mu\left(x_{1}\right) \quad \geq \cdots \geq \mu\left(x_{n}\right)$.

Axiom 1:Sp $p_{S}(\{x\})=1$.

If $\mu$ is a singleton, then $\mu\left(x_{1}\right)=1$ and $\mu\left(x_{j}\right)=0$ for all $j \neq 1$.

The steps of the algorithm are computed as follows $\Im\left(x_{1}\right):=\mu\left(x_{1}\right)=1$.

In further steps, the algorithm runs as follows for $k=2 \ldots n \operatorname{Max}\left\{T\left(1, S\left(x_{1}, x_{k}\right)\right), T\left(0, S\left(x_{2}, x_{k}\right)\right), \ldots, T(0, S\right.$ $\left.\left.\left(x_{k-1}, x_{k}\right)\right)\right\}=T\left(1, S\left(x_{1}, x_{k}\right)\right)=S\left(x_{1}, x_{k}\right) \geq \mu\left(x_{k}\right)=$ 0

So $x_{1} \succeq x_{k}$ for all $k \neq 1$, then $\Im=x_{1}$ and $S p_{S}(\mu)=$ $S p(\Im)=1$ because $\Im$ is a singleton.

Axiom 2: $S p_{S}(\emptyset)=0$. 
The steps of the algorithm are computed as follows $\Im\left(x_{1}\right):=$ $\mu\left(x_{1}\right)=0$.

In further steps, the algorithm runs as follows for $k=2 \ldots n$

$\operatorname{Max}\left\{T\left(0, S\left(x_{1}, x_{k}\right)\right), T\left(0, S\left(x_{2}, x_{k}\right)\right), \ldots\right.$,

$\left.T\left(0, S\left(x_{k-1}, x_{k}\right)\right)\right\}=0=\mu\left(x_{k}\right)=0, \operatorname{so} \Im\left(x_{k}\right):=\mu\left(x_{k}\right)=0$.

So $\Im=\emptyset$ and $S p_{S}(\mu)=S p(\emptyset)=0$.

Axiom 3: $S p_{I d}(\mu)=S p(\mu)$.

When the T-indistinguishability is the identity, Id, the steps of the algorithm are computed as follows:

$$
\Im\left(x_{1}\right):=\mu\left(x_{1}\right) .
$$

In further steps, the algorithm runs as follows for $k=2 \ldots n$

$\operatorname{Max}\left\{T\left(\mu\left(x_{1}\right), 0\right), T\left(\mu\left(x_{2}\right), 0\right), \ldots, T\left(\mu\left(x_{k-1}\right), 0\right)\right\}=0 \leq \mu\left(x_{k}\right)$

so $\Im\left(x_{k}\right):=\mu\left(x_{k}\right)$ for all $k$ and $S p_{I d}(\mu)=S p(\mu)$.

Axiom 4: $S_{p s}(\mu) \geq S p(\mu)$.

In the first step $\Im\left(x_{1}\right)=\mu\left(x_{1}\right)$. If $\mu$ is normal then $\Im$ is also normal. Also, $\Im \subseteq \subseteq \mu$, and then $S p_{S}(\mu)=S p(\Im)$ $S p(\mu)$.

\section{EXAMPLES}

\section{A. Example 6.1}

Given the min-indistinguishability $\mathrm{S}$ in example 4.1, the min-inference independent set of the fuzzy set $\mu=\left\{1 / x_{1}, 0.7 / x_{2}, 0.5 / x_{3}, 0.2 / x_{4}, 0 / x_{5}\right\}$ is $\{\Im=$ $\left.1 / x_{1}, 0 / x_{2}, 0.5 / x_{3}, 0 / x_{4}, 0 / x_{5}\right\}$. So, the measure of specificity of $\mu$ under the min-indistinguishability $S$ is the measure of specificity of the fuzzy set $\Im$.

1) If the given measure of specificity of fuzzy sets is the linear measure [4] defined as

$$
S p(\mu)=\mu\left(x_{1}\right)-\sum_{j=2}^{d} w_{j} \mu\left(x_{j}\right)
$$

with a weight $w_{2}=1$, then the measure of specificity of $\mu$ under $S$ is computed as follows: $S p_{S}(\mu)=S p(\Im)=$ $S p\left(1 / x_{1}, 0 / x_{2}, 0.5 / x_{3}, 0 / x_{4}, 0 / x_{5}\right)=1-0.5=0.5$.

Note that $S p(\mu)=1-0.7=0.3<S p_{S}(\mu)$.

2) If the measure of specificity of fuzzy sets is

$$
S p(\mu)=\mu\left(x_{1}\right) \quad \prod_{j=2}^{d}\left(1-w_{j} \mu\left(x_{j}\right)\right)
$$

with a weight $w_{2}=1$, then the measure of specificity of $\mu$ under $S$ is: $S p_{S}(\mu)=S p(\Im)=$ $S p\left(1 / x_{1}, 0 / x_{2}, 0.5 / x_{3}, 0 / x_{4}, 0 / x_{5}\right)=1 \times(1--0,5)=$ 0.5 .

Note that $S p(\mu)=1 \times(1--0.7)=0.3<S p_{S}(\mu)$.

\section{B. Example 6.2}

Given the prod-indistinguishability $S$ in Example 4.2, the fuzzy Prod-inference independent set of the fuzzy set $\mu=\left\{1 / x_{1}, 0.7 / x_{2}, 0.5 / x_{3}, 0.2 / x_{4}, 0 / x_{5}\right\}$ is $\{\Im=$ $\left.1 / x_{1}, 0.7 / x_{2}, 0.5 / x_{3}, 0 / x_{4}, 0 / x_{5}\right\}$.

a) If the measure of specificity of fuzzy sets is the linear measure of specificity (2) with a weight $w_{2}=1$, then the measure of specificity of $\mu$ under $S$ is

$$
\begin{array}{r}
S p_{S}(\mu)=S p(\Im)=S p\left(1 / x_{1}, 0.7 / x_{2}, 0.5 / x_{3}, 0 / x_{4}, 0 / x_{5}\right) \\
=1-0.7=0.3 .
\end{array}
$$

If the chosen weights are $w_{2}=0.5, w_{3}=0.5$, then the measure of specificity of $\mu$ under $S$ is $S p_{S}(\mu)=$ $S p(\Im)=S p\left(1 / x_{1}, 0.7 / x_{2}, 0.5 / x_{3}, 0 / x_{4}, 0 / x_{5}\right)=1-$ $0.35-0.25=0.4$

b) If the measure of specificity of fuzzy sets is computed by using (3) with weights $w_{2}=w_{3}=0.5$, then the measure of specificity of $\mu$ under $S$ is $S p_{S}(\mu)=$ $S p(\Im)=S p\left(1 / x_{1}, 0.7 / x_{2}, 0.5 / x_{3}, 0 / x_{4}, 0 / x_{5}\right)=$ $1 \times(1--0.35) \times(1--0.25)=1 \times 0.65 \times 0.75=0.4875$.

To solve the problem of making a useful choice of an element in $X$, the prod-indistinguishability $S$ is telling us that any choice is similar to $x_{1}, x_{2}$ or $x_{3}$ and then the choice is easier.

\section{CONCLUSION}

In previous literature on measures of specificity it was not solved the problem of computing measures of specificity under the knowledge of a T-indistinguishability. A simpler problem is solved in [5] under a similarity, whose $\alpha$-cuts are classical equivalence relations, by integrating the inverse of the cardinality of their classes. However this method is not valid for any T-indistinguishability, because its $\alpha$-cuts are not necessarily equivalence relations.

This paper solves this problem by giving the axioms of measures of specificity under T-indistinguishabilities and an algorithm to compute the inference independent set $\Im$ from a fuzzy set and a T-indistinguishability. The measures of specificity under T-indistinguishabilities of a fuzzy set $\mu$ is computed as the measure of specificity of the fuzzy iinference independent set $\Im$. It is proved that the new proposed measure of specificity under T-indistinguishabilities satisfies the four axioms of measures of specificity under T-indistinguishabilities.

Two examples are provided. The first one (4.1 and 6.1) is using a similarity, and the results are as in [5]. The second example (4.2 and 6.2) is the first approach in literature that uses a T-indistinguishability that is not a similarity.

The "jacket problem" is solved for any T-indistinguishability and a methodological tool is provided to be used in many applications.

\section{REFERENCES}

[1] L. Garmendia, "Contribución al estudio de las medidas en la lógica borrosa: Condicionalidad, especificidad y transitividad," Ph.D. dissertation, Univ. Politécnica de Madrid. E.T.S.I. Caminos, Canales y Puertos, Madrid, Spain, 2001.

[2] L. Garmendia, R. R Yager, E. Trillas, and A. Salvador, "On t-norms based measures of specificity," Fuzzy Sets Syst., vol. 133, pp. 237-248, 2003.

[3] George J. Klir and Bo Yuan, Fuzzy Sets and Fuzzy Logic: Theory and Applications. Upper Saddle River, NJ: Prentice-Hall, 1995.

[4] R. R Yager, "Ordinal measures of specificity," Int. J. Gen. Syst., vol. 17, pp. $57-72,1990$ 
[5] R. R Yager, "Similarity based measures of specificity," Int. J. Gen. Syst., vol. 19, pp. 91-106, 1991.

[6] L. A. Zadeh, "Similarity relations and fuzzy orderings," Inform. Sci., vol. 3, pp. 177-200, 1971.

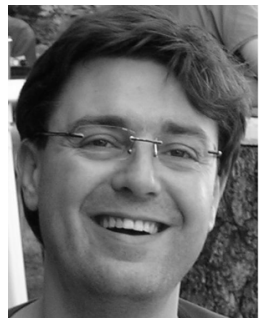

L. Garmendia received the Mathematics studies degree, with a speciality in computer science, from the Complutense University of Madrid, Spain, in 1995, and the Ph.D. degree in applied ,athematics from the Technical University of Madrid (UPM), Spain, in 2001.

He was with IBM Learning Services, Spain, form 1998 to 2003, teaching database administration, applications, and system database performance in mainframe environments. He also worked at an IBM residency in Almaden Research Center, California, and at the International Technical Support Organization (ITSO) to write an IBM Redbook. He has been teaching computer programming and mathematics in several universities in Madrid: Alfonso X el Sabio, Carlos III, Technical University of Madrid, and currently at the Computing Faculty in the Complutense University of Madrid, where he also teaches Ph.D. courses on approximate reasoning with uncertainty. Since 1995, he has participated in most of the international conferences on fuzzy logic, writing 28 papers in proceedings, nine papers in international journals, three books, and two chapters of books. He has been a member of FLAT (today EUSFLAT) since 1995, and has participated in nine research projects and directed one research project on useful measures in fuzzy logic.

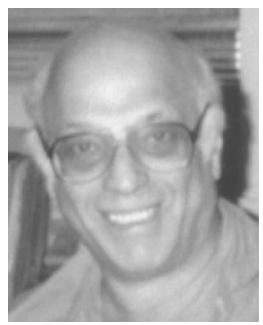

R. R. Yager received the undergraduate degree from the City College of New York and the Ph. D. degree from the Polytechnic University of New York, Brooklyn.

He has served at the National Science Foundation as Program Director in the Information Sciences Program. He was a NASA/Stanford Visiting Fellow, as well as a Research Associate, at the University of California, Berkeley. He has served as a Lecturer at NATO Advanced Study Institutes. Currently, he is Director of the Machine Intelligence Institute and Professor of Information and Decision Technologies at Iona College, New Rochelle, NY. He has published over 500 articles and fifteen books. In addition to his pioneering work in the area of fuzzy logic, he has made fundamental contributions in decision making under uncertainty and the fusion of information. His current research interests include the development of technologies for a more intelligent Internet (e-commerce, data mining, information retrieval) aggregation theory, decision making under uncertainty, and higher order information fusion.
Dr. Yager is Editor-in-Chief of the International Journal of Intelligent Systems. He serves on the Editorial Board of a number of journals, including the IEEE TRANSACTIONS ON FUZZY SYSTEMS, Neural Networks, Data Mining and Knowledge Discovery, the IEEE TRANSACTIONS ON INTELLIGENT SYSTEMS, Fuzzy Sets and Systems, the Journal of Approximate Reasoning, and the International Journal of General Systems. He is one of the Cofounders of the Conference on Information Processing and the Management of Uncertainty (IPMU). He is a Fellow of the New York Academy of Sciences and the Fuzzy Systems Association.

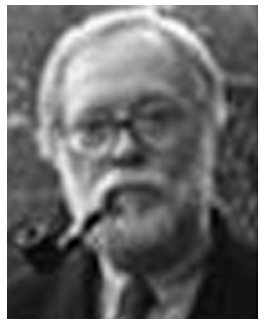

E. Trillas received the $\mathrm{Ph} . \mathrm{D}$. degree in sciences from the University of Barcelona, Barcelona, Spain, in 1972.

From 1974 to 1985, he taught at the Technical University of Barcelona, where he took several academic responsibilities in the Computing and Architecture Faculties and as Vice Rector of the university extensions as Vice Rector of studies. During the late 1980s, he was President of the Spanish Council of Science Investigations (CSIC). In the 1990s, he was the General Director of the INTA (Spanish Institute of Space and Flying Techniques) and General Secretary of the Spanish Plan of Investigations and Developments. Since 1990, he has been a Distinguished Professor with the Computing Science and Artificial intelligence Department of the Faculty of Computing at he Technical University of Madrid, Spain. He is the author of many publications, and has directed $16 \mathrm{Ph} . \mathrm{D}$. projects.

Dr. Trillas is a usual collaborator on international conferences and scientific committees, with many medals and awards, including the three most recognized awards in fuzzy logic: the Pioneer Award of the European Society For Fuzzy Logic and Technologies (EUSFLAT), Fellow of the International Fuzzy System Association (IFSA), and the most recent Fuzzy Systems Pioneer Award.

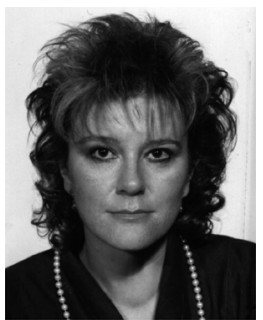

A. Salvador received the mathematics studies degree from the Complutense University of Madrid, Madrid, Spain, in 1969, and the Ph.D. degree from the Alcala de Henares University, Spain, in 1988, where she was a pioneer on fuzzy algebra research.

She taught mathematics at the Complutense University of Madrid from 1969 to 1971, and preuniversitary teacher mathematics from 1971 to 1990. During this period, she wrote 59 books on how to teach mathematics and several biographies of female mathematicians. She has been teaching applied mathematics at the Technical University of Madrid since 1990. She has been a member of FLAT (today EUSFLAT) since 1995. She has written 70 papers in conference proceedings and international journals, and has collaborated on the organization of 21 congresses. She has participated in 30 research projects. 